\title{
First neutrino interaction candidates at the LHC
}

Henso Abreu, ${ }^{1}$ Yoav Afik, ${ }^{1}$ Claire Antel, ${ }^{2}$ Jason Arakawa, ${ }^{3}$ Akitaka Ariga, ${ }^{4,5}$ Tomoko Ariga $\odot,{ }^{6, *}$ Florian Bernlochner, ${ }^{7}$ Tobias Boeckh, ${ }^{7}$ Jamie Boyd, ${ }^{8}$ Lydia Brenner, ${ }^{8}$ Franck Cadoux, ${ }^{2}$ David W. Casper, ${ }^{3}$ Charlotte Cavanagh, ${ }^{9}$ Francesco Cerutti, ${ }^{8}$ Xin Chen, ${ }^{10}$ Andrea Coccaro, ${ }^{11}$ Monica D'Onofrio, ${ }^{9}$ Candan Dozen, ${ }^{10}$ Yannick Favre, ${ }^{2}$ Deion Fellers,${ }^{12}$ Jonathan L. Feng, ${ }^{3}$ Didier Ferrere, ${ }^{2}$ Stephen Gibson, ${ }^{13}$ Sergio Gonzalez-Sevilla, ${ }^{2}$ Carl Gwilliam, ${ }^{9}$ Shih-Chieh Hsu, ${ }^{14}$ Zhen $\mathrm{Hu},{ }^{10}$ Giuseppe Iacobucci, ${ }^{2}$ Tomohiro Inada, ${ }^{10}$ Ahmed Ismail, ${ }^{15}$ Sune Jakobsen, ${ }^{8}$ Enrique Kajomovitz, ${ }^{1}$ Felix Kling,${ }^{16}$ Umut Kose, ${ }^{8}$ Susanne Kuehn, ${ }^{8}$ Helena Lefebvre, ${ }^{13}$ Lorne Levinson, ${ }^{17} \mathrm{Ke} \mathrm{Li},{ }^{14}$ Jinfeng Liu, ${ }^{10}$ Chiara Magliocca, ${ }^{2}$ Josh McFayden, ${ }^{18}$ Sam Meehan, ${ }^{8}$ Dimitar Mladenov, ${ }^{8}$ Mitsuhiro Nakamura, ${ }^{19}$ Toshiyuki Nakano, ${ }^{19}$ Marzio Nessi, ${ }^{8}$ Friedemann Neuhaus, ${ }^{20}$ Laurie Nevay, ${ }^{13}$ Hidetoshi Otono, ${ }^{6}$ Carlo Pandini, ${ }^{2}$ Hao Pang, ${ }_{10}^{10}$ Lorenzo Paolozzi, ${ }^{2}$

Brian Petersen, ${ }^{8}$ Francesco Pietropaolo, ${ }^{8}$ Markus Prim, ${ }^{7}$ Michaela Queitsch-Maitland, ${ }^{8}$ Filippo Resnati ${ }^{8}$ Hiroki Rokujo, ${ }^{19}$ Marta Sabaté-Gilarte, ${ }^{8}$ Jakob Salfeld-Nebgen, ${ }^{8}$ Osamu Sato ${ }_{19}$ Paola Scampoli, ${ }^{4,21}$ Kristof Schmieden, ${ }^{20}$ Matthias Schott, ${ }^{20}$ Anna Sfyrla, ${ }^{2}$ Savannah Shively, ${ }^{3}$ John Spencer, ${ }^{14}$ Yosuke Takubo, ${ }^{22}$ Ondrej Theiner, ${ }^{2}$ Eric Torrence, ${ }^{12}$ Sebastian Trojanowski, ${ }^{23}$ Serhan Tufanli, ${ }^{8}$ Benedikt Vormwald, ${ }^{8}$ Di Wang, ${ }^{10}$ and Gang Zhang ${ }^{10}$

(FASER Collaboration)

\footnotetext{
${ }^{1}$ Department of Physics and Astronomy, Technion-Israel Institute of Technology, Haifa 32000, Israel

${ }^{2}$ Département de Physique Nucléaire et Corpusculaire, University of Geneva, CH-1211 Geneva 4, Switzerland

${ }^{3}$ Department of Physics and Astronomy, University of California, Irvine, California 92697-4575, USA

${ }^{4}$ Albert Einstein Center for Fundamental Physics, Laboratory for High Energy Physics, University of Bern, Sidlerstrasse 5, CH-3012 Bern, Switzerland

${ }^{5}$ Department of Physics, Chiba University, 1-33 Yayoi-cho Inage-ku, Chiba 263-8522, Japan

${ }^{6}$ Kyushu University, Nishi-ku, 819-0395 Fukuoka, Japan

${ }^{7}$ Universität Bonn, Regina-Pacis-Weg 3, D-53113 Bonn, Germany

${ }^{8}$ CERN, CH-1211 Geneva 23, Switzerland

${ }^{9}$ University of Liverpool, Liverpool L69 3BX, United Kingdom

${ }^{10}$ Department of Physics, Tsinghua University, Beijing, China

${ }^{11}$ INFN Sezione di Genova, Via Dodecaneso, 33-16146, Genova, Italy

${ }^{12}$ University of Oregon, Eugene, Oregon 97403, USA

${ }^{13}$ Royal Holloway, University of London, Egham, TW20 OEX, United Kingdom

${ }^{14}$ Department of Physics, University of Washington, P.O. Box 351560, Seattle, Washington 98195-1560, USA

${ }^{15}$ Oklahoma State University, Stillwater, Oklahoma 74078-3072, USA

${ }^{16}$ SLAC National Accelerator Laboratory, 2575 Sand Hill Road, Menlo Park, California 94025, USA

${ }^{17}$ Department of Particle Physics and Astrophysics, Weizmann Institute of Science, Rehovot 76100, Israel

${ }^{18}$ Department of Physics \& Astronomy, University of Sussex, Sussex House, Falmer, Brighton, BN1 9RH, United Kingdom

${ }^{19}$ Nagoya University, Furo-cho, Chikusa-ku, Nagoya 464-8602, Japan

${ }^{20}$ Institut für Physik, Universitt Mainz, Mainz, Germany

${ }^{21}$ Dipartimento di Fisica “Ettore Pancini," Università di Napoli Federico II,

Complesso Universitario di Monte S. Angelo, I-80126 Napoli, Italy

${ }^{22}$ Institute of Particle and Nuclear Study, KEK, Oho 1-1, Tsukuba, Ibaraki 305-0801, Japan

${ }^{23}$ Astrocent, Nicolaus Copernicus Astronomical Center Polish Academy of Sciences, ul. Bartycka 18, 00-716 Warsaw, Poland
}

(Received 13 May 2021; revised 18 July 2021; accepted 26 October 2021; published 24 November 2021)

\footnotetext{
*Corresponding author. tomoko.ariga@cern.ch

Published by the American Physical Society under the terms of the Creative Commons Attribution 4.0 International license. Further distribution of this work must maintain attribution to the author(s) and the published article's title, journal citation, and DOI. Funded by SCOAP.
} 
FASER $\nu$ at the CERN Large Hadron Collider (LHC) is designed to directly detect collider neutrinos for the first time and study their cross sections at $\mathrm{TeV}$ energies, where no such measurements currently exist. In 2018, a pilot detector employing emulsion films was installed in the far-forward region of ATLAS, $480 \mathrm{~m}$ from the interaction point, and collected $12.2 \mathrm{fb}^{-1}$ of proton-proton collision data at a center-ofmass energy of $13 \mathrm{TeV}$. We describe the analysis of this pilot run data and the observation of the first neutrino interaction candidates at the LHC. This milestone paves the way for high-energy neutrino measurements at current and future colliders.

DOI: 10.1103/PhysRevD.104.L091101

\section{INTRODUCTION}

There has been a long-standing interest in detecting neutrinos produced at colliders [1-6], but to date no collider neutrino has ever been directly detected. Proton-proton $(p p)$ collisions at a center-of-mass energy of $14 \mathrm{TeV}$ during LHC Run-3, with an expected integrated luminosity of $150 \mathrm{fb}^{-1}$, will produce a high-intensity beam of $\mathcal{O}\left(10^{12}\right)$ neutrinos in the far-forward direction with mean interaction energy of about $1 \mathrm{TeV}$. FASER $\nu$ [7] is designed to detect these neutrinos and study their properties. The detector was approved in December 2019, will be installed $480 \mathrm{~m}$ downstream of the ATLAS interaction point (IP) in 2021, and will take data starting in 2022. Deployment on the beam collision axis maximizes the flux of all three neutrino flavors and allows FASER $\nu$ to measure their interaction cross sections in the currently unexplored $\mathrm{TeV}$ energy range. For electron and tau neutrinos, these measurements will extend existing cross section measurements to significantly higher energies. For muon neutrinos, they will probe the gap between accelerator measurements $\left(E_{\nu}<360 \mathrm{GeV}\right)$ [8] and IceCube data $\left(E_{\nu}>6.3 \mathrm{TeV}\right)$ [9]. Following the FASER $\nu$ approval, the SND@LHC experiment [10] designed to measure neutrinos at the LHC in a complementary rapidity region to FASER $\nu$ was approved in 2021.

In 2018, we performed a pilot run in the LHC tunnel to measure background and demonstrate neutrino detection at the LHC for the first time. Although the pilot detector lacked the ability to identify muons, given its depth of only $0.6 \lambda_{\text {int }}$, much shorter than the $8 \lambda_{\text {int }}$ of the full FASER $\nu$ detector, these data have aided reconstruction tool development and proven the feasibility of neutrino measurements in this experimental environment. Here we report the detection of neutrino interaction candidates in the pilot run data.

\section{THE PILOT RUN IN LHC RUN-2}

In 2018, we installed a 29-kg pilot detector in the TI18 tunnel, $480 \mathrm{~m}$ from the ATLAS IP, to measure neutrino interactions. With respect to the ATLAS IP, the TI18 tunnel is symmetric to TI12, where FASER $\nu$ will be located in LHC Run-3. Previously, we reported charged particle flux measurements made with other emulsion detectors installed in the TI12 and TI18 tunnels in 2018 [11]. Here we focus on the pilot detector.

The pilot detector is divided into a 14-kg module with 101 1-mm-thick lead plates and a $15-\mathrm{kg}$ module with 120 0.5 -mm-thick tungsten plates, each containing the corresponding number of emulsion films [12], as shown in Fig. 1. These emulsion films and target plates were spare parts of the NA65/DsTau experiment [13]. Each module was vacuum-packed to preserve the alignment between films and placed in a 21-cm-deep acrylic chamber. The transverse dimensions of the plates and films are $12.5 \mathrm{~cm}$ wide and $10 \mathrm{~cm}$ high.

The beam collision axis in TI18 was mapped out by the CERN survey team with mm precision. The two modules were placed side by side with the collision axis passing between them. The estimated uncertainty of the detector position is $\pm 1 \mathrm{~cm}$ in both dimensions transverse to the collision axis. An integrated luminosity of $12.2 \mathrm{fb}^{-1}$ with an uncertainty of $2 \%$ measured by the ATLAS experiment $[14,15]$ was collected during four weeks of data taking from September to October with $p p$ collisions at $13-\mathrm{TeV}$ center-of-mass energy. The beam half-crossing-angle was about $150 \mu \mathrm{rad}$ vertically upward, which moves the collision axis at the FASER location upward by $\sim 7 \mathrm{~cm}$. The detector temperature was stable at $17.94{ }^{\circ} \mathrm{C}$ with a standard deviation of $0.07^{\circ} \mathrm{C}[16]$. Temperature stability is important to avoid displacement of the emulsion films and metallic plates and to ensure good alignment. The entire lead module and $15 \%$ of the tungsten module were used in the following analysis; the remaining spare films in the tungsten module had data quality problems.
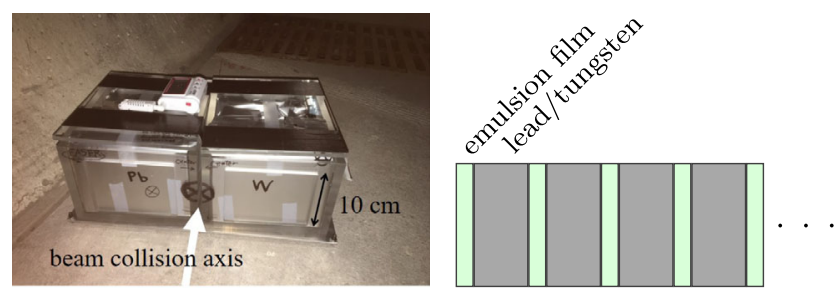

FIG. 1. Structure of the pilot emulsion detector. Metallic plates (1-mm-thick lead or $0.5-\mathrm{mm}$-thick tungsten) are interleaved with $0.3-\mathrm{mm}$-thick emulsion films. Only a schematic slice of the detector is depicted. 


\section{SIMULATION}

Neutrinos produced in the forward direction at the LHC originate from the decay of hadrons, mainly pions, kaons, and $D$ mesons. Light hadron production is simulated using the EPOS-LHC [17], QGSJET-II-04 [18], SIBYLL 2.3C [19,20], and DPMJET-III 2017.1 [21,22] simulation tools, as implemented in the CRMC [23] package, while heavy hadron production is simulated using SIBYLL 2.3C, DPMJET-III 2017.1, and PYTHIA $8.2[24,25]$ with the Monash tune [26]. Longlived hadrons are then propagated through the forward LHC beam pipe and magnetic fields using a dedicated simulation [27] implemented as a RIVET module [28] using the geometry and beam optics for Run-2 as modeled by BDSIM [29]. We use 13-TeV collision energy and a beam half-crossing-angle of $150 \mu \mathrm{rad}$ vertically upward. The hadrons are decayed at multiple locations along their trajectory according to decay branching fractions and kinematics provided by PYTHIA 8.2, and the spectra of neutrinos passing through the pilot detector are tabulated. We then use GENIE $[30,31]$ with the configuration outlined in Ref. [7] to simulate neutrino interactions.

The dominant source of background to neutrino interactions in the pilot run is inelastic interactions of neutral hadrons produced in muon photonuclear interactions upstream of the detector. The flux and spectrum of muons have been estimated by the CERN sources, targets, and interactions group, which performed FLUKA simulations [32,33]; $10^{8} p p$ collisions were simulated, and muons were propagated to the location when the beam collision axis left the concrete lining of the LHC tunnel ( $409 \mathrm{~m}$ from the IP) by the FLUKA simulation. The estimated muon flux as a function of energy at the 409-m position is shown in Fig. 2. The expected uncertainty on the FLUKA flux is of the order of $50 \%$. The muons were further propagated through $67 \mathrm{~m}$ of rock to reach close to the pilot detector by a GEANT4 simulation [34]. The expected muon fluxes at the pilot detector position are $9.4 \times 10^{3} \mu^{-}$and $3.9 \times 10^{3} \mu^{+} / \mathrm{cm}^{2} / \mathrm{fb}^{-1}$ for $E_{\mu}>100 \mathrm{GeV}$,

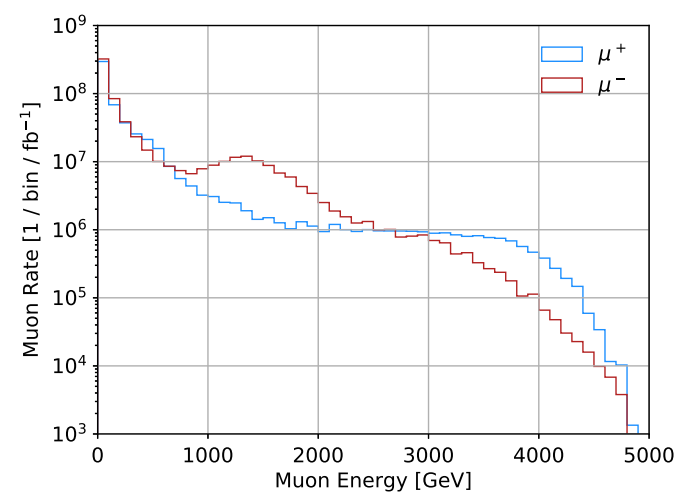

FIG. 2. The muon flux as a function of energy at $409 \mathrm{~m}$ from the IP, as estimated by FLUKA. Muons entering $1 \times 1 \mathrm{~m}^{2}$ around the collision axis are shown. and $1.5 \times 10^{4} \mu^{-}$and $9.3 \times 10^{3} \mu^{+} / \mathrm{cm}^{2} / \mathrm{fb}^{-1}$ for $E_{\mu}>10 \mathrm{GeV}$.

To simulate background hadron production and interactions, GEANT4 simulations of muons passing through the last $8 \mathrm{~m}$ of rock before reaching the pilot detector were performed. Neutral hadrons produced in the last $2 \mathrm{~m}$ in front of the pilot detector are the most relevant, because those produced further upstream are absorbed in the rock before reaching the detector. The average rock density around CERN is measured to be about $2.5 \mathrm{~g} / \mathrm{cm}^{3}$ [35]. To reproduce this density, the rock was modeled as a mixture of $41 \% \mathrm{CaCO}_{3}$ and $59 \% \mathrm{SiO}_{2} .10^{9}$ negative muons and $10^{9}$ positive muons were simulated. Kaons and neutrons are the relevant secondary neutral hadrons produced, with a small contribution from $\Lambda$ baryons. Table I shows the production rates of neutral hadrons per incident muon for negative muons and positive muons. Muon-induced neutral hadrons have a steeply falling energy spectrum; a $10-\mathrm{GeV}$ minimum energy threshold is applied to the simulation, since lowerenergy hadrons cannot satisfy the vertex reconstruction criteria used in our analysis. Neutral hadron interactions with the pilot detector were simulated by GEANT4 using the FTFP_BERT and QGSP_BERT physics lists, which correspond to different high-energy hadronic models [36].

\section{DATA ANALYSIS}

The data analysis is based on the readout of the full emulsion films by the hyper-track selector (HTS) system [37] with a readout speed of $0.45 \mathrm{~m}^{2} / \mathrm{h} /$ layer. The HTS identifies track segments ("microtracks") in the top and bottom emulsion layers of each film. A "base track" is formed by linking the two microtracks on a film. Each base track provides a 3D coordinate, 3D vector, and energy deposit $(d E / d x)$ estimator.

Data processing is broken up into subvolumes with a maximum size of $2 \times 2 \mathrm{~cm}^{2} \times 25$ emulsion films. A preliminary alignment between each two consecutive films (position shifts and gap) is obtained using recorded tracks. To further improve the tracking resolution, an additional alignment calibration is applied by selecting tracks crossing many plates. Track reconstruction then links base tracks on

TABLE I. The production rates of neutral hadrons per incident muon with an energy threshold of $10 \mathrm{GeV}$. The difference between $\mu^{-}$and $\mu^{+}$is mainly due to the difference in the energy spectra.

\begin{tabular}{lcc}
\hline \hline & Negative muons & Positive muons \\
\hline$K_{L}$ & $3.3 \times 10^{-5}$ & $9.4 \times 10^{-6}$ \\
$K_{S}$ & $8.0 \times 10^{-6}$ & $2.3 \times 10^{-6}$ \\
$n$ & $2.6 \times 10^{-5}$ & $7.7 \times 10^{-6}$ \\
$\bar{n}$ & $1.1 \times 10^{-5}$ & $3.2 \times 10^{-6}$ \\
$\Lambda$ & $3.5 \times 10^{-6}$ & $1.8 \times 10^{-6}$ \\
$\bar{\Lambda}$ & $2.8 \times 10^{-6}$ & $8.7 \times 10^{-7}$ \\
\hline \hline
\end{tabular}


TABLE II. Efficiencies for selecting interaction vertices for the signal and background. The background efficiencies are estimated for interactions of neutral hadrons with energy $>10 \mathrm{GeV}$. The statistical uncertainties are below 0.001 for all cases.

\begin{tabular}{|c|c|c|c|c|}
\hline & & \multicolumn{3}{|c|}{ Background } \\
\hline \multicolumn{2}{|c|}{ Signal } & & FTFP_BERT & QGSP_BERT \\
\hline$\nu_{e}$ & 0.490 & $K_{L}$ & 0.017 & 0.015 \\
\hline$\overline{\nu_{e}}$ & 0.343 & $K_{S}$ & 0.037 & 0.031 \\
\hline$\nu_{\mu}$ & 0.377 & $n$ & 0.011 & 0.012 \\
\hline$\overline{\nu_{\mu}}$ & 0.266 & $\bar{n}$ & 0.013 & 0.013 \\
\hline$\nu_{\tau}$ & 0.454 & $\Lambda$ & 0.020 & 0.021 \\
\hline$\overline{\nu_{\tau}}$ & 0.368 & $\bar{\Lambda}$ & 0.018 & 0.018 \\
\hline
\end{tabular}

different films by correlating their positions and angles. The track density in the data sample, some $10^{5} / \mathrm{cm}^{2}$ in a small angular space of $10 \mathrm{mrad}$, is relatively high compared to other emulsion experiments. A dedicated tracking algorithm for high density environments [13] is therefore employed on top of the software framework developed for the OPERA experiment [38].

The majority of the tracks observed in the detector are expected to be background muons and related electromagnetic showers. These background charged particles were analyzed using ten emulsion films in the lead module. The position resolution in this dataset is $0.5 \mu \mathrm{m}$ and the angular resolution is $0.2 \mathrm{mrad}$. The observed angular distribution is peaked in the direction of the ATLAS IP. The angular spread of the peak is $2.3 \mathrm{mrad}$ horizontally and $1.1 \mathrm{mrad}$ vertically. The spatial distribution was uniform within the detector volume. Track detection efficiency was determined from the single film efficiency measured for tracks penetrating ten plates. The estimated track detection efficiency for this flux measurement was $(88 \pm 5) \%$. After the efficiency correction, the charged particle flux within $10 \mathrm{mrad}$ of the angular peak, which is dominated by energetic muons, is $(1.7 \pm 0.1) \times$ $10^{4}$ tracks $/ \mathrm{cm}^{2} / \mathrm{fb}^{-1}$ normalized by luminosity. This result is consistent with the values previously reported by other detectors $[7,16]$ and close to the FLUKA prediction of $2.5 \times 10^{4}$ tracks $/ \mathrm{cm}^{2} / \mathrm{fb}^{-1}$ for $E_{\mu}>10 \mathrm{GeV}$.

For the neutrino analysis using reconstructed tracks passing through at least three plates, vertex reconstruction was performed by searching for converging patterns of tracks with a minimum distance within $5 \mu \mathrm{m}$. Converging patterns with five or more tracks were then identified as vertices, rejecting the photon background. Collimation cuts were applied to these vertices to select high-energy interactions and suppress neutral hadron backgrounds: (1) The number of tracks with $\tan \theta \leq 0.1$ with respect to the beam direction is required to be five or more, and (2) the number of tracks with $\tan \theta>0.1$ with respect to the beam direction is required to be four or less. Vertices are categorized as charged or neutral based on the presence or absence, respectively, of charged parent tracks. A looser track selection is used for the charged parent track search with a higher track detection efficiency of $99.8_{-0.3}^{+0.1} \%$. The background from charged vertices being reconstructed as neutral vertices is therefore negligible. The estimated selection efficiencies for neutrino signal and neutral hadron background vertices are shown in Table II. Signal classification is not performed in this analysis, and interactions of all neutrino flavors are combined in the data.

The fiducial volume is defined by removing seven films upstream, five films downstream, and $5 \mathrm{~mm}$ from the sides of the detector, corresponding to an 11- $\mathrm{kg}$ target mass. Within this volume, 18 neutral vertices passed the vertex selection criteria. Figure 3 shows two selected neutral vertices in lead, with 11 and nine associated charged particles, respectively.

The expected number of neutrino signal vertices after all selections is $3.3_{-0.9}^{+1.7}$, dominated by muon neutrino interactions. The uncertainty reflects only the range of predictions obtained from different Monte Carlo simulations. The expected numbers of neutral hadron background vertices are 11.0 (FTFP_BERT) and 10.1 (QGSP_BERT). Since the
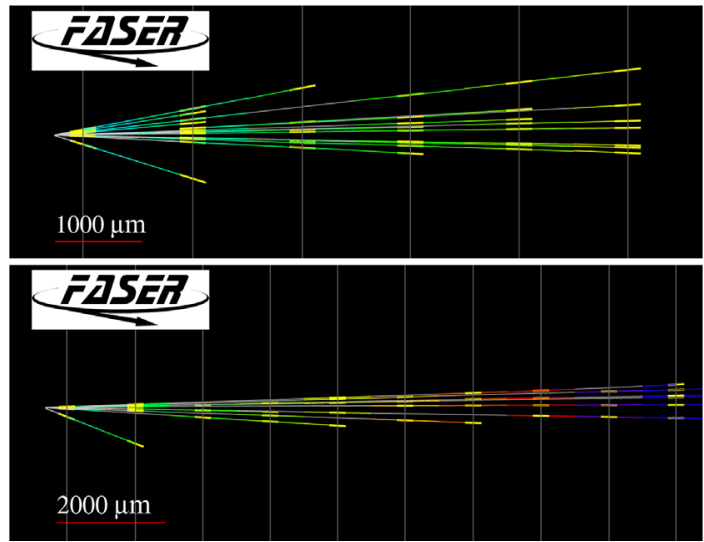

FIG. 3. Event displays of two of the neutral vertices in the $y z$ projection longitudinal to the beam direction (left) and in the view transverse to the beam direction (right).

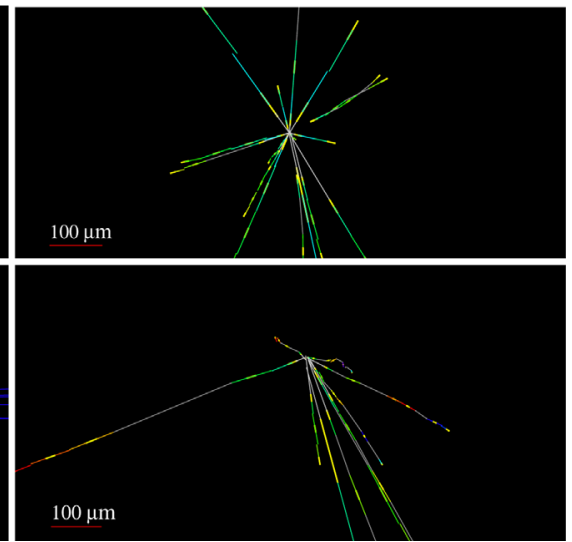


difference of the two physics lists is not significant, FTFP_BERT is used in the following analysis.

To validate the interaction features for the multivariate analysis described later, charged vertices (vertices with charged parent tracks attached), which simulation studies show also originate from muons, were analyzed. Although our muon flux measurement is close to the FLUKA prediction, no estimate of the uncertainty of the muon energy spectrum is available. The expected number of charged vertices satisfying the selection criteria is 115.4 (40.4 charged hadron interactions and 75.0 muon interactions) compared to the 78 charged vertices observed in the data.

Since the pilot detector lacked the ability to identify muons, which could allow a clean separation of neutrino charged-current and neutral hadron vertices, we introduced the following multivariate approach as a much less powerful, but necessary, alternative. A multivariate discriminant based on a boosted decision tree (BDT) algorithm has been developed to distinguish neutrino signal from neutral hadron background in the neutral vertex sample. The BDT was implemented using the Toolkit for Multivariate Data Analysis [39] and trained with Monte Carlo events passing the vertex selection criteria. To define input variables for the BDT analysis, we selected high-energy interactions and checked the momentum balance of particles in the transverse plane. Using track angles with respect to the collision axis $(\theta)$, the following variables were defined: (1) the number of tracks with $\tan \theta \leq 0.1$ with respect to the beam direction, (2) the number of tracks with $0.1<\tan \theta \leq 0.3$ with respect to the beam direction,
(3) the absolute value of the vector sum of transverse angles calculated considering all the tracks as unit vectors in the plane transverse to the beam direction $\left(a_{\text {sum }}\right) ;(4)$ for each track in the event, we calculate the mean azimuthal angle between that track and all others, in the plane transverse to the beam direction, and then take the maximum value in the event $\left(\phi_{\text {mean }}\right)$; (5) for each track in the event, we calculate the ratio of the number of tracks with azimuthal opening angle $\leq 90^{\circ}$ and $>90^{\circ}$ in the plane transverse to the beam direction, and then take the maximum value in the event $(r)$. The expected distributions of the input variables for the neutrino signal and for the neutral hadron background compared with the data are shown in Fig. 4.

The charged vertices mentioned above can be used to validate the modeling of the BDT input variables in simulated data. Figure 5 shows that the BDT inputs for simulated charged hadron and muon interactions agree well with the charged vertex data.

The BDT estimator values for the data and simulated neutral vertices are compared in Fig. 6. Here, the normalization of the signal and background distributions is freely fitted to the data, resulting in the best fit values of 6.1 and 11.9 events, respectively. The vertices shown in Fig. 3 correspond to the first and second largest BDT values. An excess of events over the background expectation is observed at high BDT estimator, which is in agreement with the background-plus-signal hypothesis. A hypothesis test using the ROOSTATS tool implemented in the CERN ROOT framework [40] is carried out on the binned BDT estimator distribution. The background-only hypothesis is
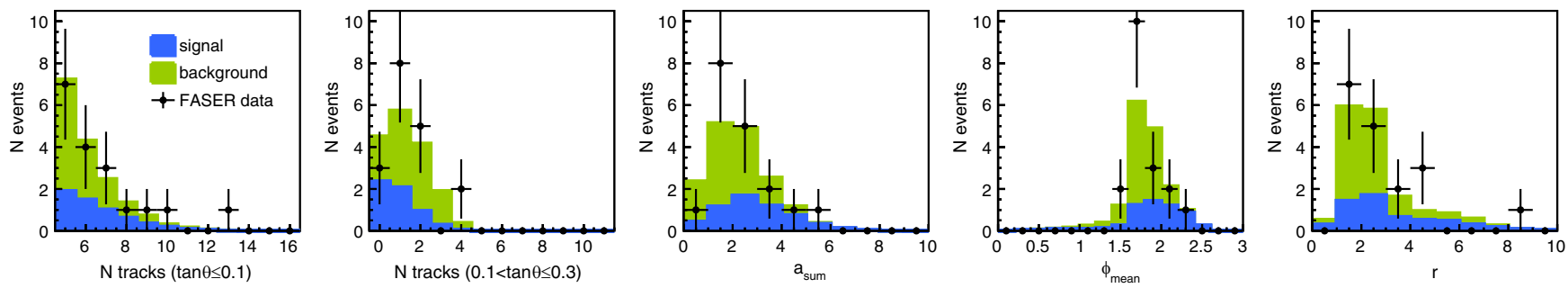

FIG. 4. Monte Carlo simulation distributions of the BDT input variables for the neutrino signal and neutral hadron background. The observed neutral vertices in the data sample are shown in black. The Monte Carlo simulation distributions are normalized to $12.2 \mathrm{fb}^{-1}$.
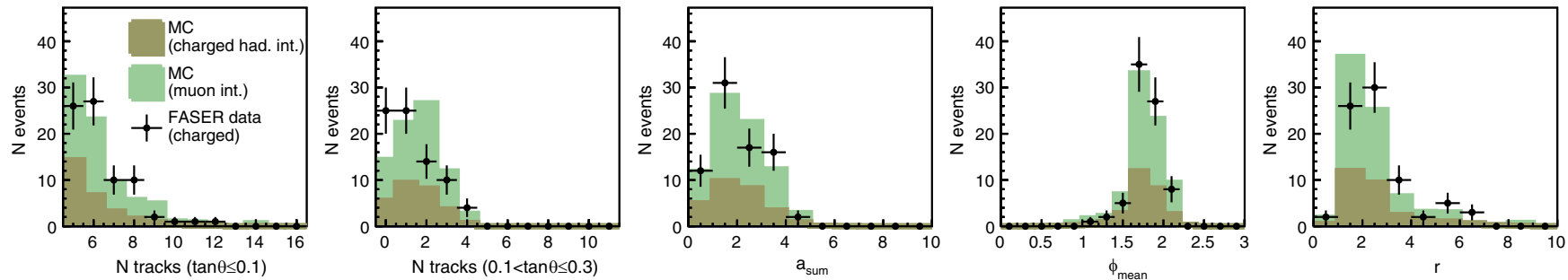

FIG. 5. Monte Carlo simulation distributions of the BDT input variables for charged hadron interactions and muon interactions. The observed charged vertices in the data sample are shown in black. The Monte Carlo simulation distributions are normalized to the data to compare the shapes. 


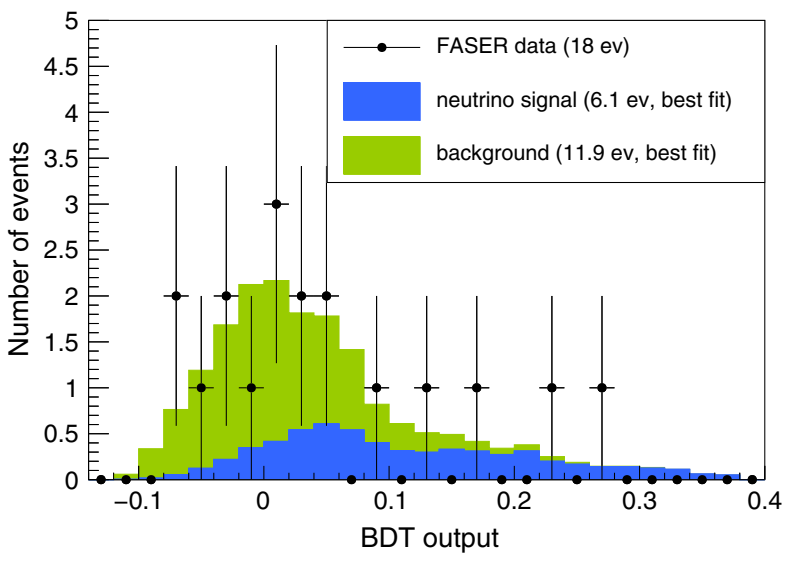

FIG. 6. The BDT outputs of the observed neutral vertices, and the expected signal and background distributions (stacked) fitted to the data. Higher BDT output values are associated with neutrinolike vertex features.

disfavored with a statistical significance of $2.7 \sigma$. The expected significance is estimated with pseudo experiments with the signal expectation of 3.3 events to be $1.7 \sigma$.

A systematic uncertainty related to the shape of the BDT distribution for neutrino events was estimated by varying the generator used for neutrino production and redoing the analysis. This resulted in a small ( $<0.2$ events) change in the fitted neutrino yield.

Systematic uncertainties on the shape of the background BDT distribution were also evaluated by varying the shape of the muon energy distribution, by varying the modeling of the photonuclear interactions in the rock that produce the background neutral hadrons from the incoming muons, and varying the physics lists for the hadron interactions. These effects can change the energy and type of the neutral hadron interacting in the detector and therefore can influence the shape of the BDT distributions. The muon distribution was scaled up and down by a factor $(1+E / 3 \mathrm{TeV})$ distorting the spectrum as a function of energy, and the analysis repeated. Fitting the data with the updated background BDT shapes changed the fitted neutrino yield by 0.1 events. In addition, the analysis was repeated using FLUKA to model the production of neutral hadrons instead of GEANT4; this leads to a change in the fitted neutrino yield of 0.1 events. Also, the analysis was repeated using the physics list QGSP_BERT to model the hadron interactions instead of FTFP_BERT; this leads to a change in the fitted neutrino yield of 0.1 events.

\section{CONCLUSIONS AND OUTLOOK}

A search for neutrino interactions is presented based on a small emulsion detector installed at the LHC in 2018. We observe the first candidate vertices consistent with neutrino interactions at the LHC. A $2.7 \sigma$ excess of neutrinolike signal above muon-induced backgrounds is measured. These results demonstrate FASER $\nu$ 's ability to detect neutrinos at the LHC and pave the way for future collider neutrino experiments.

We are currently preparing for data taking in LHC Run-3. With a deeper detector and lepton identification capability, FASER $\nu$ will perform better than the pilot run detector. In addition, the FASER spectrometer will measure the muon flux, reducing uncertainties on background estimates. In the 2022-2024 run, we expect to collect 10, 000 flavor-tagged charged-current neutrino interactions.

\section{ACKNOWLEDGMENTS}

We thank CERN for the excellent performance of the LHC and the technical and administrative staff members at all FASER institutions. We also gratefully acknowledge invaluable assistance from many groups at CERN, particularly the Physics Beyond Colliders study group, the ATLAS Collaboration for providing the luminosity value, the NA65/DsTau Collaboration for providing their spare emulsion films and tungsten plates for this measurement, and Masahiro Komatsu for useful discussions. This work was supported in part by Heising-Simons Foundation Grants No. 2018-1135, No. 2019-1179, and No. 20201840, Simons Foundation Grant No. 623683, and the Department of Energy Grant No. DE-SC0016013. This work was supported by JSPS KAKENHI Grants No. JP19H01909, No. JP20H01919, No. JP20K04004, and No. JP20K23373, a research grant from the Mitsubishi Foundation, and the joint research program of the Institute of Materials and Systems for Sustainability.
[1] A. De Rujula and R. Ruckl, Neutrino and muon physics in the collider mode of future accelerators, in Proceedings of the SSC Workshop: Superconducting Super Collider Fixed Target Physics (1984), pp. 571-596, http://cds.cern.ch/record/1337790.

[2] K. Winter, Detection of the tau-neutrino at the LHC, in Proceedings of the ECFA Large Hadron Collider
(LHC) Workshop: Physics and Instrumentation (1990), pp. 37-49, http://cds.cern.ch/record/220304.

[3] F. Vannucci, Neutrino physics at LHC/SSC, in Proceedings of the 4th International Symposium on Neutrino Telescopes (1993), pp. 57-68, http://cds.cern.ch/record/253670.

[4] A. De Rujula, E. Fernandez, and J. Gomez-Cadenas, Neutrino fluxes at future hadron colliders, Nucl. Phys. B405, 80 (1993). 
[5] H. Park, The estimation of neutrino fluxes produced by proton-proton collisions at $\sqrt{s}=14 \mathrm{TeV}$ of the LHC, J. High Energy Phys. 10 (2011) 092.

[6] J. L. Feng, I. Galon, F. Kling, and S. Trojanowski, ForwArd Search ExpeRiment at the LHC, Phys. Rev. D 97, 035001 (2018).

[7] H. Abreu et al. (FASER Collaboration), Detecting and studying high-energy collider neutrinos with FASER at the LHC, Eur. Phys. J. C 80, 61 (2020).

[8] P. A. Zyla et al. (Particle Data Group), Review of particle physics, Prog. Theor. Exp. Phys. 2020, 083C01 (2020).

[9] M. Aartsen et al. (IceCube Collaboration), Measurement of the multi-TeV neutrino cross section with IceCube using Earth absorption, Nature (London) 551, 596 (2017).

[10] C. Ahdida et al., SND@LHC - Scattering and Neutrino Detector at the LHC, CERN Report No. CERN-LHCC2021-003, 2021.

[11] H. Abreu et al. (FASER Collaboration), Technical Proposal: FASERnu, CERN Report No. CERN-LHCC-2019-017, 2019.

[12] A. Ariga, T. Ariga, G. D. Lellis, A. Ereditato, and K. Niwa, Nuclear Emulsions (Springer International Publishing, Cham, 2020), pp. 383-438, https://doi.org/10.1007/978-3030-35318-6_9.

[13] S. Aoki et al. (DsTau Collaboration), DsTau: Study of tau neutrino production with $400 \mathrm{GeV}$ protons from the CERNSPS, J. High Energy Phys. 01 (2020) 033.

[14] ATLAS Collaboration, Luminosity determination in $p p$ collisions at $\sqrt{s}=13 \mathrm{TeV}$ using the ATLAS detector at the LHC, CERN Report No. ATLAS-CONF-2019-021, 2019.

[15] G. Avoni et al., The new LUCID-2 detector for luminosity measurement and monitoring in ATLAS, J. Instrum. 13, P07017 (2018).

[16] A. Ariga et al. (FASER Collaboration), Technical Proposal for FASER: ForwArd Search ExpeRiment at the LHC, CERN Report No. CERN-LHCC-2018-036, 2018.

[17] T. Pierog, I. Karpenko, J. M. Katzy, E. Yatsenko, and K. Werner, EPOS LHC: Test of collective hadronization with data measured at the CERN Large Hadron Collider, Phys. Rev. C 92, 034906 (2015).

[18] S. Ostapchenko, Monte Carlo treatment of hadronic interactions in enhanced Pomeron scheme: I. QGSJET-II model, Phys. Rev. D 83, 014018 (2011).

[19] E.-J. Ahn, R. Engel, T. K. Gaisser, P. Lipari, and T. Stanev, Cosmic ray interaction event generator SIBYLL 2.1, Phys. Rev. D 80, 094003 (2009).

[20] F. Riehn, R. Engel, A. Fedynitch, T. K. Gaisser, and T. Stanev, A new version of the event generator Sibyll, Proc. Sci., ICRC2015 (2016) 558 [arXiv:1510.00568].

[21] S. Roesler, R. Engel, and J. Ranft, The Monte Carlo event generator DPMJET-III, in Proceedings of the International Conference on Advanced Monte Carlo for Radiation Physics, Particle Transport Simulation and Applications
(MC 2000), Lisbon, Portugal, 2000, arXiv:hep-ph/0012252, https://doi.org/10.1007/978-3-642-18211-2_166.

[22] A. Fedynitch, Cascade equations and hadronic interactions at very high energies, CERN Report No. CERN-THESIS2015-371, 2015, https://cds.cern.ch/record/2231593.

[23] C. Baus, T. Pierog, and R. Ulrich, Cosmic ray Monte Carlo (CRMC), https://web.ikp.kit.edu/rulrich/crmc.html.

[24] T. Sjostrand, S. Mrenna, and P. Z. Skands, PYTHIA 6.4 physics and manual, J. High Energy Phys. 05 (2006) 026.

[25] T. Sjstrand, S. Ask, J. R. Christiansen, R. Corke, N. Desai, P. Ilten, S. Mrenna, S. Prestel, C. O. Rasmussen, and P.Z. Skands, An introduction to PYTHIA 8.2, Comput. Phys. Commun. 191, 159 (2015).

[26] P. Skands, S. Carrazza, and J. Rojo, Tuning PYTHIA 8.1: the Monash 2013 Tune, Eur. Phys. J. C 74, 3024 (2014).

[27] F. Kling, Forward neutrino fluxes and simulation at the LHC (to be published).

[28] C. Bierlich et al., Robust independent validation of experiment and theory: RIVET version 3, SciPost Phys. 8, 026 (2020).

[29] L. J. Nevay et al., BDSIM: An accelerator tracking code with particle-matter interactions, Comput. Phys. Commun. 252, 107200 (2020).

[30] C. Andreopoulos et al., The GENIE neutrino Monte Carlo generator, Nucl. Instrum. Methods Phys. Res., Sect. A 614, 87 (2010).

[31] C. Andreopoulos, C. Barry, S. Dytman, H. Gallagher, T. Golan, R. Hatcher, G. Perdue, and J. Yarba, The GENIE neutrino Monte Carlo generator: Physics and user manual, arXiv:1510.05494.

[32] A. Ferrari, P. R. Sala, A. Fasso, and J. Ranft, FlukA: A multi-particle transport code, 2005.

[33] G. Battistoni et al., Overview of the FLUKA code, Ann. Nucl. Energy 82, 10 (2015).

[34] S. Agostinelli et al., GEANT4-Simulation toolkit, Nucl. Instrum. Methods Phys. Res., Sect. A 506, 250 (2003).

[35] E. J. Fern, V. Di Murro, K. Soga, Z. Li, L. Scibile, and J. A. Osborne, Geotechnical characterisation of a weak sedimentary rock mass at CERN, Geneva, Tunn. Undergr. Space Technol. 77, 249 (2018).

[36] J. Allison et al., Recent developments in GEANT4, Nucl. Instrum. Methods Phys. Res., Sect. A 835, 186 (2016).

[37] M. Yoshimoto, T. Nakano, R. Komatani, and H. Kawahara, Hyper-track selector nuclear emulsion readout system aimed at scanning an area of one thousand square meters, Prog. Theor. Exp. Phys. 2017, 103 H01 (2017).

[38] V. Tyukov, I. Kreslo, Y. Petukhov, and G. Sirri, The FEDRA-Framework for emulsion data reconstruction and analysis in the OPERA experiment, Nucl. Instrum. Methods Phys. Res., Sect. A 559, 103 (2006).

[39] A. Hoecker et al., TMVA-Toolkit for multivariate data analysis, arXiv:physics/0703039.

[40] F. Rademakers et al., ROOT-PROJECT/ROOT: v6.16/02, 2018, https://doi.org/10.5281/zenodo.1292566. 